\title{
Polinômios Ortogonais no Círculo Unitário : Coeficientes de Verblunsky Associados a Sequências Reais de Sinal Alternante
}

\section{Cleonice F. Bracciali ${ }^{1}$}

Departamento de Matemática Aplicada, UNESP, São José do Rio Preto, SP Jairo S. Silva ${ }^{2}$

Pós-Graduação em Matemática, UNESP, São José do Rio Preto, SP

\section{A. Sri Ranga ${ }^{3}$}

Departamento de Matemática Aplicada, UNESP, São José do Rio Preto, SP

Daniel O. Veronese ${ }^{4}$

Pós-Graduação em Matemática, UNESP, São José do Rio Preto, SP

\begin{abstract}
Resumo. Recentemente, foi mostrado em [5] que associado ao par de sequências reais $\left\{\left\{c_{n}\right\}_{n=1}^{\infty},\left\{d_{n}\right\}_{n=1}^{\infty}\right\}$, com $\left\{d_{n}\right\}_{n=1}^{\infty}$ uma sequência encadeada positiva, existe uma única medida de probabilidade não trivial $\mu$ no círculo unitário. Mostrou-se também que os coeficientes de Verblunsky $\left\{\alpha_{n}\right\}_{n=0}^{\infty}$, associados aos polinômios ortogonais com respeito a $\mu$, podem ser relacionados diretamente com tais sequências. Neste trabalho, consideramos esta relação e suas consequências quando impomos uma restrição de sinal sobre a sequência $\left\{c_{n}\right\}_{n=1}^{\infty}$. Precisamente, quando a sequência $\left\{c_{n}\right\}_{n=1}^{\infty}$ tem uma propriedade de sinal alternante, usamos informações sobre os zeros de certos polinômios para-ortogonais para estimar o suporte da medida associada.
\end{abstract}

Palavras-chave. Polinômios para-ortogonais, Medidas de probabilidade, Sequências encadeadas positivas, Sequências de sinal alternante.

\section{Introdução}

Polinômios ortogonais no círculo unitário (OPUC) tem sido comumente conhecido como polinômios de Szegő em homenagem a Gábor Szegő que os apresentou na primeira metade do século 20. Por causa de suas aplicações em regras de quadratura, processamento de sinais, teoria espectral e muitos outros tópicos, estes polinômios têm recebido muita

\footnotetext{
${ }^{1}$ cleonice@ibilce.unesp.br

2 jairomath@hotmail.com

${ }^{3}$ ranga@ibilce.unesp.br

${ }^{4}$ doveronese@yahoo.com.br
} 
atenção nos últimos anos (veja, por exemplo, $[2,6,8,11]$ ). Para textos mais recentes sobre tais polinômios nos referimos aos dois volumes de Simon $[9,10]$.

Dada uma medida de probabilidade não trivial $\mu(z)=\mu\left(e^{i \theta}\right)$ no círculo unitário $\mathcal{C}=$ $\left\{z=e^{i \theta}: 0 \leq \theta \leq 2 \pi\right\}$, a sequência associada de OPUC $\left\{S_{n}\right\}$ pode ser definida por

$$
\int_{\mathcal{C}} \bar{z}^{j} S_{n}(z) d \mu(z)=\int_{0}^{2 \pi} e^{-i j \theta} S_{n}\left(e^{i \theta}\right) d \mu\left(e^{i \theta}\right)=0, \quad 0 \leq j \leq n-1, \quad n \geq 1 .
$$

Fazendo $\kappa_{n}^{-2}=\left\|S_{n}\right\|^{2}=\int_{\mathcal{C}}\left|S_{n}(z)\right|^{2} d \mu(z)$, os polinômios ortonormais no círculo unitário são $s_{n}(z)=\kappa_{n} S_{n}(z), n \geq 0$.

Os OPUC mônicos satisfazem as relações de recorrência

$$
\begin{aligned}
& S_{n}(z)=z S_{n-1}(z)-\bar{\alpha}_{n-1} S_{n-1}^{*}(z), \\
& S_{n}(z)=\left(1-\left|\alpha_{n-1}\right|^{2}\right) z S_{n-1}(z)-\bar{\alpha}_{n-1} S_{n}^{*}(z), \quad n \geq 1,
\end{aligned}
$$

onde $\alpha_{n-1}=-\overline{S_{n}(0)}$ e $S_{n}^{*}(z)=z^{n} \overline{S_{n}(1 / \bar{z})}$ denota o polinômio revertido (recíproco) de $S_{n}(z)$. Os números $\alpha_{n}$, nos últimos anos, têm sido referidos como coeficientes Verblunsky. Sabe-se que esses coeficientes são tais que $\left|\alpha_{n}\right|<1, n \geq 0$. Além disso, os OPUC e a medida associada são completamente determinados a partir desses coeficientes (veja por exemplo [9], Theorem 1.7.11).

Foi mostrado em [5] que, dada qualquer medida de probabilidade não trivial no círculo unitário, então correspondente a esta medida, existe um par de sequências reais $\left\{c_{n}\right\}_{n=1}^{\infty} \mathrm{e}$ $\left\{d_{n}\right\}_{n=1}^{\infty}$, onde $\left\{d_{n}\right\}_{n=1}^{\infty}$ é uma sequência encadeada positiva. No Teorema 2.1 fornecemos informações completas sobre esta afirmação e sua recíproca. Para ser mais preciso, as sequências $\left\{c_{n}\right\}_{n=1}^{\infty}$ e $\left\{d_{n}\right\}_{n=1}^{\infty}$ são os coeficientes da fórmula de recorrência de três termos

$$
R_{n+1}(z)=\left[\left(1+i c_{n+1}\right) z+\left(1-i c_{n+1}\right)\right] R_{n}(z)-4 d_{n+1} z R_{n-1}(z), \quad n \geq 1,
$$

com $R_{0}(z)=1$ e $R_{1}(z)=\left(1+i c_{1}\right) z+\left(1-i c_{1}\right)$, onde

$$
R_{n}(z)=\frac{\prod_{j=1}^{n}\left[1-\tau_{j-1} \alpha_{j-1}\right]}{\prod_{j=1}^{n}\left[1-\mathcal{R} e\left(\tau_{j-1} \alpha_{j-1}\right)\right]} \frac{z S_{n}(z)-\tau_{n} S_{n}^{*}(z)}{z-1},
$$

$\operatorname{com} \tau_{n}=S_{n}(1) / S_{n}^{*}(1), n \geq 0$.

Dado o par de sequências reais $\left\{c_{n}\right\}_{n=1}^{\infty}$ e $\left\{d_{n}\right\}_{n=1}^{\infty}$, é possível recuperar a medida de probabilidade associada a este par, considerando certas funções racionais que seguem da fórmula de recorrência (2). Em [3], usando argumentos padrão envolvendo frações contínuas, expansões em séries no infinito e na origem e o teorema da Seleção de Helly, a medida associada $\mu$ é dada como um limite de uma subsequência de medidas discretas $\psi_{n}\left(e^{i \theta}\right)$ cujos pontos puros (aqueles que são diferentes de $z=1$ ) são exatamente os zeros de $R_{n}(z)$. Os resultados apresentados em [3] nos permite dar informações sobre o suporte da medida $\mu$ estudando os zeros $z_{n, j}=e^{i \theta_{n, j}}, j=1,2 \ldots, n$, de $R_{n}(z)$, ou, equivalentemente, estudando os zeros das funções $W_{n}(x)$, dadas por

$$
W_{n}(x)=2^{-n} e^{-i n \theta / 2} R_{n}\left(e^{i \theta}\right), \quad n \geq 0,
$$


onde $x=\cos (\theta / 2)$. A sequência de funções $\left\{W_{n}\right\}_{n=0}^{\infty}$ satisfaz a fórmula de recorrência de três termos (veja $[1,7])$

$$
W_{n+1}(x)=\left(x-c_{n+1} \sqrt{1-x^{2}}\right) W_{n}(x)-d_{n+1} W_{n-1}(x), \quad n \geq 1,
$$

com $W_{0}(x)=1$ e $W_{1}(x)=x-c_{1} \sqrt{1-x^{2}}$.

Em [7], prova-se que, para qualquer $n \geq 1, W_{n}$ tem exatamente $n$ zeros distintos $x_{n, j}=$ $\cos \left(\theta_{n, j} / 2\right), j=1,2 \ldots, n$, em $(-1,1)$, os quais satisfazem a propriedade de entrelaçamento

$$
-1<x_{n+1, n+1}<x_{n, n}<x_{n+1, n}<\cdots<x_{n, 1}<x_{n+1,1}<1, \quad n \geq 1,
$$

isto é, entre dois zeros consecutivos de $W_{n+1}$ existe um zero de $W_{n}$.

O objetivo deste trabalho é estudar sequências de coeficientes de Verblunsky onde a sequência relacionada $\left\{c_{n}\right\}_{n=1}^{\infty}$ tem uma restrição de sinal. Mostramos que, sobre certas condições, é possível estimar o suporte da medida associada.

\section{Resultados Preliminares}

Nesta seção, apresentamos alguns resultados sobre medidas de probabilidade não triviais e sequências encadeadas (para mais detalhes sobre sequências encadeadas veja, por exemplo, [4]). Iniciamos com um teorema estabelecido em [5] que fornece uma caracterização para medidas de probabilidade não triviais em termos de duas sequências reais.

Teorema 2.1. (a) Dada uma medida de probabilidade não trivial $\mu$ sobre o círculo unitário, então associado com esta, existe um único par de sequências reais $\left\{\left\{c_{n}\right\}_{n=1}^{\infty},\left\{d_{n}\right\}_{n=1}^{\infty}\right\}$, onde $\left\{d_{n}\right\}_{n=1}^{\infty}$ é também uma sequência encadeada positiva. Especificamente, se $\left\{\alpha_{n}\right\}_{n=0}^{\infty}$ é a sequência associada de coeficientes de Verblunsky e se a sequência $\tau_{n}$ é tal que

$$
\tau_{0}=1 \quad e \quad \tau_{n}=\tau_{n-1} \frac{1-\bar{\tau}_{n-1} \bar{\alpha}_{n-1}}{1-\tau_{n-1} \alpha_{n-1}}, \quad n \geq 1,
$$

então $m_{0}=0$,

$$
c_{n}=\frac{-\operatorname{Im}\left(\tau_{n-1} \alpha_{n-1}\right)}{1-\operatorname{Re}\left(\tau_{n-1} \alpha_{n-1}\right)} \quad \text { e } \quad m_{n}=\frac{1}{2} \frac{\left|1-\tau_{n-1} \alpha_{n-1}\right|^{2}}{\left[1-\operatorname{Re}\left(\tau_{n-1} \alpha_{n-1}\right)\right]}, \quad n \geq 1,
$$

onde $\left\{m_{n}\right\}_{n=0}^{\infty}$ é a sequência minimal de parâmetros de $\left\{d_{n}\right\}_{n=1}^{\infty}$. Além disso, a sequência maximal de parâmetros $\left\{M_{n}\right\}_{n=0}^{\infty}$ de $\left\{d_{n}\right\}_{n=1}^{\infty}$ é tal que $M_{0}$ é o valor do salto na medida em $z=1$.

(b) Reciprocamente, dado um par de sequências reais $\left\{\left\{c_{n}\right\}_{n=1}^{\infty},\left\{d_{n}\right\}_{n=1}^{\infty}\right\}$, onde $\left\{d_{n}\right\}_{n=1}^{\infty}$ é também uma sequência encadeada positiva, então, associado com este par, existe uma única medida de probabilidade não trivial $\mu$ suportada no círculo unitário. Especificamente, se $\left\{m_{n}\right\}_{n=0}^{\infty}$ é a sequência minimal de parâmetros de $\left\{d_{n}\right\}_{n=1}^{\infty}$, então $\tau_{0}=1$,

$$
\tau_{n-1} \alpha_{n-1}=\frac{1-2 m_{n}-i c_{n}}{1-i c_{n}} \quad \text { e } \quad \tau_{n}=\frac{1-i c_{n}}{1+i c_{n}} \tau_{n-1}, \quad n \geq 1 .
$$

Além disso, a medida tem um salto $M_{0}$ em $z=1$, onde $\left\{M_{n}\right\}_{n=0}^{\infty}$ é a sequência maximal de parâmetros de $\left\{d_{n}\right\}_{n=1}^{\infty}$. 
Agora, apresentamos um teorema que fornece uma relação entre os zeros dos polinômios $R_{n}(z)$ e a medida associada com o par de sequências reais $\left\{\left\{c_{n}\right\}_{n=1}^{\infty},\left\{d_{n}\right\}_{n=1}^{\infty}\right\}$. O mesmo é uma consequência imediata de resultados obtidos em [3].

Teorema 2.2. Seja $\left\{\left\{c_{n}\right\}_{n=1}^{\infty},\left\{d_{n}\right\}_{n=1}^{\infty}\right\}$ um par de sequências reais, com $\left\{d_{n}\right\}_{n=1}^{\infty}$ uma sequência encadeada positiva. Além disso, seja $R_{n}(z)$ a sequência de polinômios dada por (2) e $\mu$ a medida associada com este par de sequências. Mais ainda, suponha que os zeros de $R_{n}(z)$ estejam sobre um arco fechado $\mathcal{B}$ do círculo unitário, para todo $n \geq 1$. Então, o suporte da medida $\mu$ se encontra dentro de $\mathcal{B} \cup\{1\}$.

\section{Estimativas para o Suporte de Medidas Associadas com Sequências $\left\{c_{n}\right\}$ de Sinal Alternante}

Primeiro, estabelecemos dois lemas utilizados para obter os resultados subsequentes.

Lema 3.1. Seja $W_{n}(x)$ satisfazendo (4) e $R_{n}(z)$ satisfazendo (2). Então, as seguintes afirmações são equivalentes:

(i) $c_{n}=(-1)^{n} c, n \geq 1$ e $c \in \mathbb{R}$;

(ii) $R_{2 n}(z)$ é real e $R_{2 n+1}(z)=[(1-i c) z+(1+i c)] \widetilde{R}_{2 n}(z)$ com $\widetilde{R}_{2 n}(z)$ real para $n \geq 0$;

(iii) $W_{2 n}(x)$ é um polinômio par de grau $2 n$ e $W_{2 n+1}(x)=\left(x+c \sqrt{1-x^{2}}\right) \widetilde{W}_{2 n}(x)$ com $\widetilde{W}_{0}(x)=1$ e $\widetilde{W}_{2 n}(x)$ um polinômio par de grau $2 n$, para $n \geq 0$.

Demonstração. (i) $\Rightarrow$ (ii) Como $R_{0}(z)=1$ e $R_{1}(z)=[(1-i c) z+(1+i c)] \widetilde{R}_{0}(z)$, com $\widetilde{R}_{0}(z)=1$, o resultado ocorre se $n=0$.

Além disso, aplicando-se a relação de recorrência de três termos (2) para $R_{2}(z)$ vemos facilmente que $R_{2}(z)$ é um polinômio real. Usando novamente a relação de recorrência para $R_{3}(z)$ e a hipótese $(\mathrm{i})$, obtemos $R_{3}(z)=[(1-i c) z+(1+i c)] \widetilde{R}_{2}(z)$, com $\widetilde{R}_{2}(z)=$ $R_{2}(z)-4 d_{3} z$ um polinômio real. Assim, o resultado também ocorre se $n=1$. Continuandose da mesma maneira, o resultado segue facilmente por indução matemática.

(ii) $\Rightarrow$ (iii) Por (ii), $R_{2 n}(z)$ é real para todo $n \geq 0$, daí seu coeficiente líder $r_{2 n, 2 n}=$ $\prod_{j=1}^{2 n}\left(1+i c_{j}\right)$ também é real. Logo, $\left(1+i c_{2 n+1}\right)\left(1+i c_{2 n+2}\right) \in \mathbb{R}$, e consequentemente,

$$
c_{2 n+2}=-c_{2 n+1}, \quad n=0,1,2, \ldots .
$$

Para $n=0$, fazendo $c_{1}=-c$ temos $W_{1}(x)=\left(x+c \sqrt{1-x^{2}}\right) \widetilde{W}_{0}(x)$, com $\widetilde{W}_{0}(x)=1$. Assim, o resultado ocorre para $n=0$. Além disso, por $(7), c_{2}=c$.

Para $n=1$, usando-se a relação de recorrência de três termos (4) para $W_{2}(x)$ vemos que $W_{2}(x)$ é um polinômio par de grau 2. Novamente, pela relação de recorrência de três termos (4) para $W_{3}(x)$, temos $W_{3}(x)=\left(x-c_{3} \sqrt{1-x^{2}}\right) W_{2}(x)-d_{3} W_{1}(x)$. Por outro lado, como $\widetilde{z}=-\frac{1+i c}{1-i c}$ é um zero de $R_{3}(z)$, pela relação (3) vemos que $\widetilde{x}=-\frac{c}{\sqrt{1+c^{2}}}$ é um zero de $W_{3}(x)$. Note que $\widetilde{x}$ é também um zero de $W_{1}(x)$. Daí, $\left(\widetilde{x}-c_{3} \sqrt{1-\widetilde{x}^{2}}\right) W_{2}(\widetilde{x})=0$. Mas, pela propriedade de entrelaçamento dos zeros, $W_{2}(\widetilde{x}) \neq 0$ e portanto $\widetilde{x}-c_{3} \sqrt{1-\widetilde{x}^{2}}=0$. Assim, 
vemos que $c_{3}=-c$ e, consequentemente, $W_{3}(x)=\left(x+c \sqrt{1-x^{2}}\right) \widetilde{W}_{2}(x)$, onde $\widetilde{W}_{2}(x)=$ $W_{2}(x)-d_{3}$ é um polinômio par de grau 2. Logo o resultado ocorre para $n=1$. Usando argumentos análogos, podemos obter o resultado desejado usando indução matemática.

(iii) $\Rightarrow$ (i) Usando a hipótese (iii) e a definição de $W_{1}(x)$ é fácil ver que $c_{1}=-c$. Além disso, como $W_{2}(x)$ é par, usando (4) vemos que a função $\left(x-c_{2} \sqrt{1-x^{2}}\right) W_{1}(x)$ também é par. Daí, $c=c_{2}$. Agora, de $W_{3}(x)=\left(x+c \sqrt{1-x^{2}}\right) \widetilde{W}_{2}(x)$ e pela relação de recorrência para $W_{3}(x)$ observamos que as funções $f(x)=\left(x-c_{3} \sqrt{1-x^{2}}\right) W_{2}(x)$ e $h(x)=-d_{3}\left(x+c \sqrt{1-x^{2}}\right)$ devem ter um fator comum. Mas, sendo $W_{2}(x)$ um polinômio de grau 2 isto só pode ocorrer se $c_{3}=-c$. Continuando com o mesmo procedimento, podemos obter o resultado desejado.

Considere agora os polinômios $\hat{R}_{n}(z)$ satisfazendo

$$
\hat{R}_{n+1}(z)=\left[\left(1+i \hat{c}_{n+1}\right) z+\left(1-i \hat{c}_{n+1}\right)\right] \hat{R}_{n}(z)-4 d_{n+1} z \hat{R}_{n-1}(z), \quad n \geq 1,
$$

com $\hat{R}_{0}(z)=1, \hat{R}_{1}(z)=\left(1+i \hat{c}_{1}\right) z+\left(1-i \hat{c}_{1}\right)$ e $\hat{c}_{n}=-c_{n}$.

Lema 3.2. Seja $R_{n}(z)$ satisfazendo (2) e $\hat{R}_{n}(z)$ satisfazendo (8). Então, $R_{n}(z)=\overline{\hat{R}_{n}(\bar{z})}$, para todo $n \geq 0$.

Demonstração. A prova pode ser dada por indução matemática. Claramente, o resultado ocorre parar $n=0$ e $n=1$. Suponha que o resultado é válido para $n=0,1, \ldots, k$. Então, das relações de recorrência (2) e (8), temos

$$
\begin{aligned}
\hat{R}_{k+1}(\bar{z}) & =\left[\left(1-i c_{k+1}\right) \bar{z}+\left(1+i c_{k+1}\right)\right] \hat{R}_{k}(\bar{z})-4 d_{k+1} \bar{z} \hat{R}_{k-1}(\bar{z}) \\
& =\left[\left(1-i c_{k+1}\right) \bar{z}+\left(1+i c_{k+1}\right)\right] \overline{R_{k}(z)}-4 d_{k+1} \bar{z} \overline{R_{k-1}(z) .}
\end{aligned}
$$

Portanto, o resultado segue tomando o conjugado complexo na relação acima.

Observação 3.1. O Lema 3.2 fornece uma relação entre os zeros dos polinômios $R_{n}(z)$ e os zeros de $\hat{R}_{n}(z)$, isto é, se $z_{n, j}$ é um zero de $R_{n}(z)$ então $\bar{z}_{n, j}$ é um zero de $\hat{R}_{n}(z)$.

Agora, consideramos o problema de fornecer estimativas para o suporte de medidas cujas sequências associadas $\left\{c_{n}\right\}_{n=1}^{\infty}$ são de sinal alternante, isto é, $c_{n}=(-1)^{n} \widetilde{c}_{n}$, com $\left\{\widetilde{c}_{n}\right\}$ sendo qualquer sequência de números reais positiva (ou negativa).

Seja $\mathcal{C}_{1}=\left\{z=e^{i \theta}: 0 \leq \theta \leq \arccos \left(\frac{c^{2}-1}{c^{2}+1}\right)\right\}$ e $\mathcal{C}_{2}=\left\{z=e^{i \theta}: 2 \pi-\arccos \left(\frac{c^{2}-1}{c^{2}+1}\right) \leq \theta \leq 2 \pi\right\}$ Iniciamos com o caso $c_{n}=(-1)^{n} c$, onde $c \in \mathbb{R}^{*}$.

Teorema 3.1. Seja $\mu$ a medida de probabilidade no círculo unitário associada com o par de sequências reais $\left\{\left\{c_{n}\right\}_{n=1}^{\infty},\left\{d_{n}\right\}_{n=1}^{\infty}\right\}$ onde $c_{n}=(-1)^{n} c, c \in \mathbb{R}^{*}$ e $\left\{d_{n}\right\}_{n=1}^{\infty}$ é uma sequência encadeada positiva. Então, o suporte de $\mu$ se encontra dentro de $\mathcal{C}_{1} \cup \mathcal{C}_{2}$.

Demonstração. Sem perda de generalidade, podemos assumir que $c>0$. Pelo Teorema 2.2, basta provar que todos os zeros de $R_{n}(z)$, dado por (2), estejam sobre $\mathcal{C}_{1} \cup \mathcal{C}_{2}$. Para isto, usaremos as funções $W_{n}(x)$, dadas por (4), que estão associados aos polinômios $R_{n}(z)$.

Pelo Lema 3.1 temos $W_{2 n+1}(x)=\left(x+c \sqrt{1-x^{2}}\right) \widetilde{W}_{2 n}(x)$ com $\widetilde{W}_{2 n}(x)$ um polinômio par de grau $2 n$. Além disso, $W_{2 n}(x)$ é também um polinômio par de grau $2 n$. Isto significa 
que $\frac{-c}{\sqrt{1+c^{2}}}$ é sempre um zero de $W_{2 n+1}(x)$ e os outros $2 n$ zeros destas funções tem uma simetria em relação a origem. Da mesma forma, todos os zeros de $W_{2 n}(x)$ são simétricos com relação a origem. Portanto, desta simetria dos zeros e tendo em conta a propriedade de entrelaçamento dos zeros de $W_{n}(x)$ podemos concluir que todos os seus zeros pertencem ao conjunto $\left(-1, \frac{-c}{\sqrt{1+c^{2}}}\right] \cup\left[\frac{c}{\sqrt{1+c^{2}}}, 1\right)$. Se denotarmos os zeros de $W_{n}(x)$ por $x_{n, j}$ e os zeros de $R_{n}(z)$ por $z_{n, j}$, então estes zeros são relacionados por $x_{n, j}=\cos \left(\frac{\theta_{n, j}}{2}\right)$, onde $z_{n, j}=e^{i \theta_{n, j}}, j=1,2, \ldots, n$. Isto mostra que todos os zeros de $R_{n}(z)$ estão dentro de $\mathcal{C}_{1} \cup \mathcal{C}_{2}$.

Observação 3.2. Observe que o Teorema 3.1 fornece uma estimativa para o suporte da medida no caso de $c_{n}=(-1)^{n} \tilde{c}_{n}$, com $\tilde{c}_{n}$ sendo uma sequência constante. Usamos esta estimativa inicial para obter um resultado mais geral, dado a seguir.

Teorema 3.2. Seja $\mu$ a medida de probabilidade no círculo unitário associada com o par de sequências reais $\left\{\left\{c_{n}\right\}_{n=1}^{\infty},\left\{d_{n}\right\}_{n=1}^{\infty}\right\}$, onde $c_{n}=(-1)^{n} \tilde{c}_{n}, \tilde{c}_{n} \geq c>0, c \in \mathbb{R} e\left\{d_{n}\right\}_{n=1}^{\infty}$ é uma sequência encadeada positiva. Então, o suporte de $\mu$ se encontra dentro de $\mathcal{C}_{1} \cup \mathcal{C}_{2}$.

Demonstração. Inicialmente, note que para todo $\varepsilon \operatorname{com} 0<\varepsilon<c$, temos $\tilde{c}_{n} \geq c>c_{\varepsilon}>0$, onde $c_{\varepsilon}=c-\varepsilon$. Daí, se $x_{0}=\frac{-c_{\varepsilon}}{\sqrt{1+c_{\varepsilon}^{2}}}$ e $x_{1}=\frac{c_{\varepsilon}}{\sqrt{1+c_{\varepsilon}^{2}}}$ pode-se mostrar que, para todo $n \geq 1$,

$$
\operatorname{sign}\left(x_{0}-\tilde{c}_{n} \sqrt{1-x_{0}^{2}}\right)=\operatorname{sign}\left(x_{1}-\tilde{c}_{n} \sqrt{1-x_{1}^{2}}\right)=-1
$$

$\mathrm{e}$

$$
\operatorname{sign}\left(x_{0}+\tilde{c}_{n} \sqrt{1-x_{0}^{2}}\right)=\operatorname{sign}\left(x_{1}+\tilde{c}_{n} \sqrt{1-x_{1}^{2}}\right)=1 .
$$

Agora, notando que $\frac{-\tilde{c}_{1}}{\sqrt{1+\tilde{c}_{1}^{2}}}$ é o único zero de $W_{1}(x)$ em $(-1,1)$ e que $\tilde{c}_{1}>c$, então o resultado ocorre para $n=1$. Além disso, de $(10), \operatorname{sign}\left(W_{1}\left(x_{j}\right)\right)=1, j \in\{0,1\}$.

Assim, a partir da relação de recorrência de três termos (4) para $W_{2}(x)$ e de (9) concluímos que $\operatorname{sign}\left(W_{2}\left(x_{j}\right)\right)=-1, j \in\{0,1\}$. Suponha, por absurdo, que existe pelo menos um zero de $W_{2}(x)$ dentro do intervalo $\left(x_{0}, x_{1}\right)$, então, como $W_{2}\left(x_{0}\right)<0$ e $W_{2}\left(x_{1}\right)<$ 0 concluímos que $W_{2}(x)$ tem dois zeros em $\left(x_{0}, x_{1}\right)$. Mas isso não pode ocorrer, pois o único zero de $W_{1}(x)$ está fora de $\left(x_{0}, x_{1}\right)$ e este zero se entrelaça com os dois zeros de $W_{2}(x)$. Logo, o resultado também ocorre para $n=2$.

Novamente, da relação de recorrência para $W_{3}(x), \operatorname{sign}\left(W_{2}\left(x_{j}\right)\right)=-1, \operatorname{sign}\left(W_{1}\left(x_{j}\right)\right)=$ $1, j \in\{0,1\}$, e por (10) temos que $\operatorname{sign}\left(W_{3}\left(x_{j}\right)\right)=-1, j \in\{0,1\}$. Portanto, usando a propriedade de entrelaçamento para os zeros de $W_{3}(x)$ e $W_{2}(x)$, e o fato que não existe nenhum zero de $W_{2}(x)$ em $\left(x_{0}, x_{1}\right)$, podemos concluir que $W_{3}(x)$ não se anula em $\left(x_{0}, x_{1}\right)$.

Continuando este procedimento, por indução, pode-se mostrar que

$$
\operatorname{sign}\left(W_{n}\left(x_{j}\right)\right)=(-1)^{\lfloor n / 2\rfloor}, j \in\{0,1\}, n=0,1,2, \ldots
$$

e, pelos mesmos argumentos usados antes, $W_{n}(x)$ não se anula em $\left(x_{0}, x_{1}\right)$. Finalmente, fazendo $\varepsilon \rightarrow 0$, vemos que $W_{n}(x)$ tem todos os seus zeros em $\left(-1, \frac{-c}{\sqrt{1+c^{2}}}\right] \cup\left[\frac{c}{\sqrt{1+c^{2}}}, 1\right)$. Isto completa a prova, como no teorema anterior. 
Corolário 3.1. Seja $\mu$ a medida de probabilidade no círculo unitário associada com o par de sequências reais $\left\{\left\{c_{n}\right\}_{n=1}^{\infty},\left\{d_{n}\right\}_{n=1}^{\infty}\right\}$, onde $c_{n}=(-1)^{n} \tilde{c}_{n}, \tilde{c}_{n} \leq c<0, c \in \mathbb{R} e\left\{d_{n}\right\}_{n=1}^{\infty}$ é uma sequência encadeada positiva. Então, o suporte de $\mu$ se encontra dentro de $\mathcal{C}_{1} \cup \mathcal{C}_{2}$.

Demonstração. Segue imediatamente do Teorema 3.2 e do Lemma 3.2.

\section{Agradecimentos}

Os autores agradecem à CAPES, CNPq e FAPESP, pelo apoio financeiro para a realização deste trabalho.

\section{Referências}

[1] C. F. Bracciali, J.H. McCabe, T.E. Peréz and A. Sri Ranga, A class of orthogonal functions given by a three term recurrence formula, Math. Comp., (2015), (to appear).

[2] J. Breuer, E. Ryckman and B. Simon, Equality of the spectral and dynamical definitions of reflection, Comm. Math. Phys., vol. 295, 531-550, (2010).

[3] K. Castillo, M. S. Costa, A. Sri Ranga and D. O. Veronese, A Favard type theorem for orthogonal polynomials on the unit circle from a three term recurrence formula, J. Approx. Theory, vol. 184, 146-162, (2014).

[4] T. S. Chihara, An Introduction to Orthogonal Polynomials, Mathematics and its Applications Series, Gordon and Breach, (1978).

[5] M. S. Costa, H. M. Felix and A. Sri Ranga, Orthogonal polynomials on the unit circle and chain sequences, J. Approx. Theory, vol. 173, 14-32, (2013).

[6] M. S. Costa, E. Godoy, R. L. Lamblém and A. Sri Ranga, Basic hypergeometric functions and orthogonal Laurent polynomials, Proc. Amer. Math. Soc., vol. 140, 2075-2089, (2011).

[7] D. K. Dimitrov and A. Sri Ranga, Zeros of a family of hypergeometric para-orthogonal polynomials on the unit circle, Math. Nachr., vol. 286, 1778-1791, (2013).

[8] F. Peherstorfer, Positive trigonometric quadrature formulas and quadrature on the unit circle, Math. Comp., vol. 80, 1685-1701, (2011).

[9] B. Simon, Orthogonal Polynomials on the Unit Circle. Part 1. Classical Theory, Amer. Math. Soc. Colloq. Publ., vol. 54, part 1, (2005).

[10] B. Simon, Orthogonal Polynomials on the Unit Circle. Part 2. Spectral Theory, Amer. Math. Soc. Colloq. Publ., vol. 54, part 2, (2005).

[11] S. Tsujimoto and A. Zhedanov, Elliptic hypergeometric Laurent biorthogonal polynomials with a dense point spectrum on the unit circle, SIGMA Symmetry Integrability Geom. Methods Appl., vol. 5, 30, (2009). 